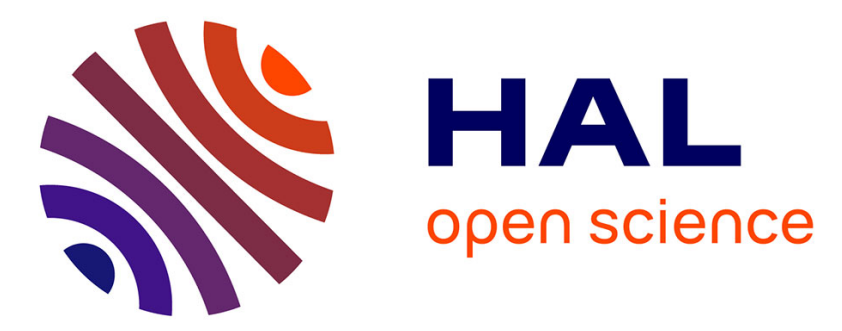

\title{
Subproblems Applied to a 3-D Magnetostatic Facet FEM Formulation
}

Anderson Santos Nunes, Patrick Dular, Olivier Chadebec, Patrick Kuo-Peng

\section{To cite this version:}

Anderson Santos Nunes, Patrick Dular, Olivier Chadebec, Patrick Kuo-Peng. Subproblems Applied to a 3-D Magnetostatic Facet FEM Formulation. IEEE Transactions on Magnetics, 2018, 54 (8), pp.1-9. 10.1109/TMAG.2018.2828786 . hal-01985331

\section{HAL Id: hal-01985331 https://hal.science/hal-01985331}

Submitted on 4 Feb 2020

HAL is a multi-disciplinary open access archive for the deposit and dissemination of scientific research documents, whether they are published or not. The documents may come from teaching and research institutions in France or abroad, or from public or private research centers.
L'archive ouverte pluridisciplinaire HAL, est destinée au dépôt et à la diffusion de documents scientifiques de niveau recherche, publiés ou non, émanant des établissements d'enseignement et de recherche français ou étrangers, des laboratoires publics ou privés. 


\title{
Subproblems applied to a 3D Magnetostatic Facet FEM formulation
}

\author{
Anderson S. Nunes ${ }^{1}$, Patrick Dular ${ }^{2}$, Olivier Chadebec ${ }^{3}$, and Patrick Kuo-Peng ${ }^{1}$ \\ ${ }^{1}$ GRUCAD/ UFSC - Universidade Federal de Santa Catarina, Florianópolis, CEP: 88040-970, Brasil \\ ${ }^{2}$ University of Liège, Department of Electrical Engineering and Computer Science, ACE, \\ Liège B-4000, Belgium \\ ${ }^{3}$ Univ. Grenoble Alpes, CNRS, Grenoble INP, G2Elab, F-38000 Grenoble, France
}

\begin{abstract}
The Facet Finite Element Method (FFEM) requires a source field solution that is commonly obtained by the Biot-Savart equation that can be time-consuming. The subproblem modeling (SPM) is applied to the FFEM in order to minimize the computational effort and time necessary to obtain the solution. The volume sources, which allow solving the Biot-Savart equation only along the active regions, is presented, as well as the Biot-Savart boundary condition correction.
\end{abstract}

Index Terms-Reluctance Network Method (RNM), Facet Finite Element Method (FFEM), Magnetic Devices, Three-Dimensional, Magnetostatics

\section{INTRODUCTION}

The Facet Finite Element Method (FFEM) has been presented as an alternative to reduce the computational time and resources when modeling magnetostatic fields in electromagnetic devices [1]-[3], because it allows an easy coupling with external magnetic networks, i.e., the Reluctance Network Method (RNM), and does not require any additional attention to deal with multiply-connected domains as in the classical magnetic scalar potential formulation.

However, the FFEM magnetostatic formulation does not take into account an electrical current source, requiring some additional computations when the discretized domains contain windings. In [4] and [5] the sources are computed considering the windings as current loops. A methodology using the electric vector potential T0 is presented in [6].

Considering all the complexities of calculating these sources, a possible solution could be to take advantage of subproblem method (SPM), which has been applied to model electromagnetic problems in a progressive way [7], allowing to benefit from a previous solution, instead of starting a new complete one for any physical variation, e.g., material properties or geometric.

This methodology has been applied to model nondestructive testing [8], to take into account the effects of air gaps [9] and in general magnetodynamics applications [10][15] applying the $\mathbf{A}$ or the $\mathbf{A}-V$ formulation. It was also used to model MEMS through an electrostatic formulation, where an unperturbed electric field is obtained and then a conductive domain is inserted as a perturbation [16].

Thus, considering that i) normally the authors start from an unperturbed solution and then apply all the perturbations necessary to take into account the physical phenomena and/or geometric characteristics in interest, ii) the source field needed in the FFEM magnetostatic formulation can be obtained with the Biot-Savart equation which can be interpreted as an unper-

Manuscript received December 1, 2012; revised August 26, 2015. Corresponding author: A. Nunes (email: nunes.anderson@gmail.com). turbed solution, and iii) all these SPM magnetic applications above cited are using $\mathbf{A}$ based formulations, this paper aims to present the SPM applied to the FFEM B-formulation, reducing the effort required to obtain the source field solution.

This is accomplished by considering the Biot-Savart solution as a first subproblem, i.e., the unperturbed one. Then, the active parts $\left(\mu_{r}>1\right)$ are added into the domain and a reaction field solution is obtained with the FFEM considering the BiotSavart source field only inside these ferromagnetic regions. The total solution is the sum of both problems (unperturbed + perturbation). This is the key point of this work, since it allows reducing the source field calculation to the active parts only, decreasing the computational time and effort.

Furthermore, it is presented how to correct the boundary condition difference between the FFEM and Biot-Savart, which allows a direct superposition of the results.

In order to validate the obtained results, two test cases are arranged. The first one is a simple limit case built based on a 2-D mesh, which was extruded creating the 3-D domain. The second test case is a representative 3-D magnetic device that is modeled using the proposed methodology. Finally, FFEM + RNM coupling [3] and the SPM are applied in this model at the same time, benefiting from the advantages of both methodologies.

Considering that this work deals with magnetostatic fields, the results obtained are compared with those calculated using the classical FEM A-formulation or with the FFEM Bformulation.

\section{MAGNETOSTATIC FFEM FORMULATION AND ITS BOUNDARY CONDITIONS}

The magnetostatic problem is defined along a bounded Euclidean domain $\Omega$ with its boundary $\Gamma=\Gamma_{H} \cup \Gamma_{B}$ (Fig. 1a). $\Omega_{a}$ can represent a perfect conductor $(\sigma \rightarrow \infty) \Omega_{p c}$, i.e., a domain where internal magnetic field is zero and results in a zero skin depth [14], [17], or a magnetic material with finite permeability $\Omega_{m} . \Omega_{s}$ is a stranded conductor in which 
the current density $\mathbf{J}_{s}$ is imposed. The interface between two different media in $\Omega$ is denoted by $\gamma$ (Fig. 1b).

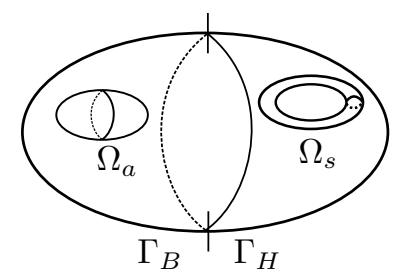

(a) Domain $\Omega$ defined by its boundary $\Gamma=\Gamma_{H} \cup \Gamma_{B}$

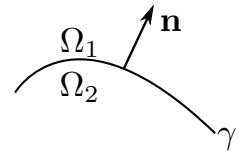

(b) Interface $\gamma$ between two media.

Fig. 1. Domain $\Omega$ and the interface $\gamma$.

The Ampère and Gauss equations, together with the magnetic constitutive relation for linear and isotropic materials, are expressed as

$$
\begin{gathered}
\operatorname{curl} \mathbf{H}=\mathbf{J}_{s} \\
\operatorname{div} \mathbf{B}=0 \\
\mathbf{B}=\mu \mathbf{H}+\mathbf{B}_{r}
\end{gathered}
$$

or

$$
\mathbf{H}=\nu \mathbf{B}+\mathbf{H}_{c}
$$

where $\mathbf{H}$ is the magnetic field, $\mathbf{B}$ is the flux density, $\mu$ is the magnetic permeability, $\nu$ is the magnetic reluctivity, $\mathbf{B}_{r}$ is the remnant flux density and $\mathbf{H}_{c}$ is the coercive field. In the SPM, the $\mathbf{B}_{r}$ or $\mathbf{H}_{c}$ can also be used as volume sources, expressing changes in the permeability.

The boundary conditions are

$$
\begin{aligned}
\mathbf{n} \times\left.\mathbf{H}\right|_{\Gamma_{H}} & =0 \\
\left.\mathbf{n} \cdot \mathbf{B}\right|_{\Gamma_{B}} & =0
\end{aligned}
$$

where $\mathbf{n}$ is the unit normal vector.

The interface conditions between two media in $\Omega, \gamma$ in Fig. 1b, are

$$
\begin{gathered}
\mathbf{n} \times\left.\left(\mathbf{H}_{1}-\mathbf{H}_{2}\right)\right|_{\gamma_{H}}=\mathbf{J}_{f} \\
\left.\mathbf{n} \cdot\left(\mathbf{B}_{1}-\mathbf{B}_{2}\right)\right|_{\gamma_{B}}=0
\end{gathered}
$$

which can be expressed through the following notation

$$
\begin{aligned}
{[\mathbf{n} \times \mathbf{H}]_{\gamma_{H}} } & =\mathbf{J}_{f} \\
{[\mathbf{n} \cdot \mathbf{B}]_{\gamma_{B}} } & =0
\end{aligned}
$$

where $\mathbf{J}_{f}$ is the surface current density. $\gamma_{H}$ and $\gamma_{B}$ are interfaces between two media considering the interface conditions for $\mathbf{H}$ and $\mathbf{B}$ fields.

\section{A. FFEM formulation}

As previously cited, this work's goal is to apply the SPM in a FFEM magnetostatic formulation, it is briefly summarized here. The details of this formulation can be found in [3].

It is established writing the total magnetic field $\mathbf{H}$ in terms of source and reaction fields, $\mathbf{H}_{s}$ and $\mathbf{H}_{r}$ respectively,

$$
\mathbf{H}=\mathbf{H}_{s}+\mathbf{H}_{r}
$$

with

$$
\operatorname{curl} \mathbf{H}_{s}=\mathbf{J}_{s}
$$

and

$$
\mathbf{H}_{r}=-\operatorname{grad} \psi
$$

where $\psi$ is the reduced (single valued) magnetic scalar potential. The source field $\mathbf{H}_{s}$ (12) is calculated by applying the Biot-Savart equation, which can be a time consuming task.

So, applying (11) and (13) in (3) leads to the strong formulation of the magnetic constitutive relation based on $\mathbf{B}$,

$$
\frac{1}{\mu} \mathbf{B}+\operatorname{grad} \psi=\mathbf{H}_{s}+\frac{1}{\mu} \mathbf{B}_{r}
$$

The weak form of (14) is

$$
\begin{array}{r}
\sum_{i=1}^{n_{f}}\left(\int_{\Omega} \frac{1}{\mu} \mathbf{B}_{j}^{\prime} \cdot \mathbf{B}_{i}^{\prime} d \Omega\right) \Phi_{i}+\psi_{a}-\psi_{b}= \\
\int_{\Omega} \mathbf{H}_{s} \cdot \mathbf{B}^{\prime} d \Omega+\int_{\Omega} \frac{1}{\mu} \mathbf{B}_{r} \cdot \mathbf{B}^{\prime} d \Omega
\end{array}
$$

with its boundary condition

$$
\left.\mathbf{n} \cdot \mathbf{B}\right|_{\Gamma_{B}}=0
$$

where $\mathbf{B}^{\prime} \in W^{2}$ are the facet shape functions [18], [19]. $\psi_{a}$ and $\psi_{b}$ are the magnetic potentials at two adjacent elements $a$ and $b$.

These basis functions have some important properties, for instance, its normal component is constant along the first order element faces. Furthermore, its flux is equal to 1 for a given face $i$

$$
\mathbf{B}_{i}^{\prime} \cdot \mathbf{n}_{i}=\frac{1}{S_{i}}
$$

and zero along the other faces. $S_{i}$ is the surface area of face $i$. Another important aspect is that its divergence in an element is equal to the inverse of the volume $V_{e}$ of this element (for first order elements):

$$
\operatorname{div} \mathbf{B}_{i}^{\prime}=\frac{1}{V_{e}}
$$

Thus, the final matrix system

$$
[\Re][\Phi]-\left[\psi_{m}\right]=\left[\psi_{0}\right]+[B r]
$$

is composed by the terms of (15): the FFEM stiffness matrix, $[\Re]$, physically known as a reluctance matrix; the unknown 
magnetic flux vector, $[\Phi]$; the averaged magnetic potential between each reluctance, $\left[\psi_{m}\right]$; the vector with all the magnetomotive force $(\mathrm{mmf})$ sources, $\left[\psi_{0}\right]$, obtained considering the magnetic field $\mathbf{H}_{s}$ calculated using Biot-Savart; and the vector which takes into account any remnant induction, $[\mathrm{Br}]$.

Since the matrix system is stated without taking into account (2), the zero divergence of $\mathbf{B}$ is enforced by (19) as a circuit system, where the Kirchhoff's current law is imposed. It suggests that the solution can be obtained by the use of a OD circuit solver, as presented in [4], [20], [21].

As aforementioned, this formulation is detailed in [3].

\section{B. The $\mathbf{n} \times\left.\mathbf{H}\right|_{\Gamma_{H}}=0$ boundary condition}

Going a step forward in the boundary conditions analysis, besides the $\left.\mathbf{n} \cdot \mathbf{B}\right|_{\Gamma_{B}}=0$ (16), it is also possible to apply $\mathbf{n} \times\left.\mathbf{H}\right|_{\Gamma_{H}}=0$. Considering that (19) is solved in the light of the circuit formalism, $\mathbf{n} \times\left.\mathbf{H}\right|_{\Gamma_{H}}=0$ is achieved by connecting all the elements along $\Gamma_{H}$ with an external node $P$ (Fig. 2), which consists in imposing a constant magnetic scalar potential on $\Gamma_{H}$, as

$$
\mathbf{n} \times\left.\mathbf{H}\right|_{\Gamma_{H}}=-\mathbf{n} \times\left.\operatorname{grad} \psi\right|_{\Gamma_{H}}=0
$$

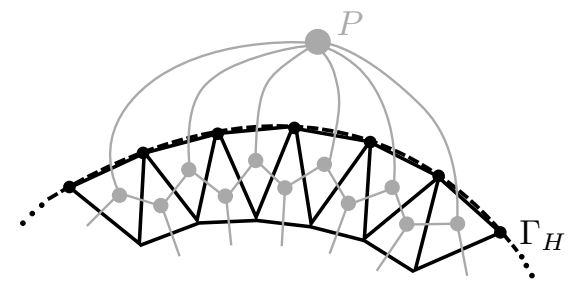

Fig. 2. Boundary condition $\mathbf{n} \times\left.\mathbf{H}\right|_{\Gamma_{H}}=0$.

If node $P$ is not connected to any other external branch, like presented in (Fig. 2), it implies a zero total magnetic flux along this surface.

This condition was discussed in our previous paper [3], but this connection is also used to impose one external flux density in the model, i.e., $\left.\mathbf{n} \cdot \mathbf{B}\right|_{\Gamma_{B}}$.

\section{Normal flux density $\left(\left.\mathbf{n} \cdot \mathbf{B}\right|_{\Gamma_{B}}\right)$ source}

Considering the FFEM circuit formalism, this source can be implemented defining flux sources along each face $i$ along $\Gamma_{B}$, i.e., one flux source at each branch connecting the face $i$ to the node $P$ shown in Fig. 2. The flux values are given by

$$
\Phi_{i}=\int_{\Gamma_{i}} \mathbf{n} \cdot \mathbf{B}_{i} d \Gamma_{i}\left(i=1 \ldots, n_{f \Gamma}\right)
$$

where $n_{f \Gamma}$ is the number of element faces along $\Gamma$ and $\mathbf{B}_{i}$ is the flux density field along each face.

This is not a time-consuming process, since the integration given in (21) is based on the Gauss points of those 2-D elements along $\Gamma$.

\section{DEFINITION OF THE SUBPROBLEMS}

Once the key concepts necessary to define the SPM in the light of the FFEM (circuit formalism) are above presented, it is possible to describe the SPM chain.

It is based on a sequence of subproblems, considering the idea of the source and reaction fields, but only calculating the source field along specific parts of the domain.

In this case, a sequence of two problems, $p$ and $q$, is presented. In problem $p$ an unperturbed field is obtained with Biot-Savart. In problem $q$, the source field is obtained, also with Biot-Savart, and the reaction field is solved with the FFEM. Then, both results are simply superposed, resulting in the total solution $(p+q)$. Depending on the case, the first and consequently the sequence of the problems can vary. Here problem $p$ is always the Biot-Savart solution and problem $q$ can differ depending on the application.

\section{A. Inductor alone}

The Biot-Savart field calculation in subproblem $p$ is obtained in $\Omega$ considering only the conductors, $\Omega_{s, p}$, where $Q \in \Omega_{p}$ is the calculation point, $P \in \Omega_{s, p}$ is the integration point and $\mathbf{r}_{P Q}$ is the position vector, with the current density $\mathbf{J}_{s}$ (Fig. 3).

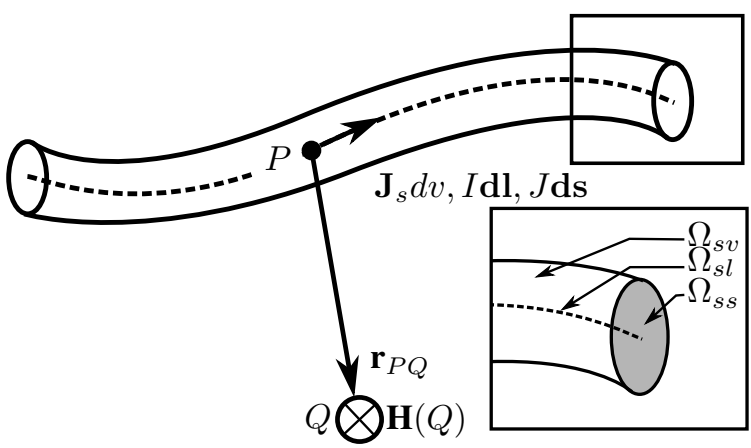

Fig. 3. Integration domain for the Biot-Savart equation.

For 3-D cases, the source field can be obtained through

$$
\mathbf{H}_{(Q)}=\frac{1}{4 \pi} \int_{\Omega_{s v}} \frac{\mathbf{J}_{(P)} \times \mathbf{r}_{P Q}}{\left|\mathbf{r}_{P Q}\right|^{3}} d \Omega_{s v}
$$

Moreover, for some specific cases where the aspect ratio between the entire domain and conductor cross-section dimensions is high, $\Omega_{s, p}$ can be simplified by its average line, not requiring a 3-D but an 1-D mesh which could represent computational advantages. In this case, the source field is obtained using

$$
\mathbf{H}_{(Q)}=\frac{I}{4 \pi} \int_{\Omega_{s l}} \frac{\mathbf{d l}_{(P)} \times \mathbf{r}_{P Q}}{\left|\mathbf{r}_{P Q}\right|^{3}} d \Omega_{s l}
$$

In case of 2-D symmetries, (22) can be re-written as

$$
\mathbf{H}_{(Q)}=\frac{J}{2 \pi} \int_{\Omega_{s s}} \frac{\mathbf{d} \mathbf{s}_{(P)} \times \mathbf{r}_{P Q}}{\left|\mathbf{r}_{P Q}\right|^{2}} d \Omega_{s s}
$$

The integration process of (22), (23) or (24) along $\Omega_{s, p}$ can be done based on a non-conforming mesh in $\Omega$, allowing the 
utilization of higher order isoparametric elements, for instance, increasing the accuracy of the source field, mainly when $\Omega_{s, p}$ contains curved regions.

The source field can be calculated normalizing the current in the conductors as $1 \mathrm{~A}$, for instance, and multiplying the field by the real currents afterwards. Also, as the conductors are immersed in a region with $\mu_{r}=1$, it is possible to explore symmetries, which can be lost in the complete problem [13].

\section{B. Correction of the boundary condition not imposed by the} Biot-Savart equation

The Biot-Savart solution, Section III-A, does not impose the boundary condition $\left.\mathbf{n} \cdot \mathbf{B}\right|_{\Gamma_{B}}=0$ along the boundaries as the FFEM does. So, a simple superposition of results, i.e., problem $p$ (Biot-savart) + problem $q$ (FFEM), might cause some differences in the total solution. This problem is also verified when using the classical FEM with $\mathbf{A}$ formulation, because it also imposes $\left.\mathbf{n} \cdot \mathbf{B}\right|_{\Gamma_{B}}=0$, meaning that this correction has to be applied there, as well.

It can be solved canceling the component $\mathbf{n} \cdot \mathbf{B}_{p}$ along the outer surface of $\Omega, \Gamma$, using the $\left.\mathbf{n} \cdot \mathbf{B}\right|_{\Gamma_{B}}$ surface sources presented in Section II-C, with $\left.\mathbf{n} \cdot \mathbf{B}_{q}\right|_{\Gamma}=-\left.\mathbf{n} \cdot \mathbf{B}_{p}\right|_{\Gamma}$. As mentioned in Section II-C, it is a light process since it depends on a 2-D integration along those element faces which comprise the surface. In addition, this step can be included in a subsequent problem, $q$.

\section{Perfect conductor boundary condition}

Other application of the methodology explained in Section III-B, i.e., the implementation of $\left.\mathbf{n} \cdot \mathbf{B}_{q}\right|_{\Gamma}=-\left.\mathbf{n} \cdot \mathbf{B}_{p}\right|_{\Gamma}$, is the limit case of a perfect conductor. Furthermore, it is a limit case that, in the light of the FFEM, applies a $\left.\mathbf{n} \cdot \mathbf{B}\right|_{\Gamma}$ field along the boundary as described in Section II-C. So, it appears as an interesting case to be analyzed.

The internal magnetic field of a perfect conductor, denoted by $\Omega_{p c} \in \Omega$ and its boundary $\gamma_{p c}$, is zero and results in a zero skin depth. This behavior is treated removing $\Omega_{p c}$ from $\Omega$ and fixing a zero total magnetic flux along its boundaries, $\gamma_{p c}$. So, assuming that there is no discontinuity along $\gamma_{p c}$ in the total solution, as in (10), it is possible to perturb the problem $p$, solved with Biot-Savart (Section III-A), defining a problem $q$ as

$$
\left[\mathbf{n} \cdot \mathbf{B}_{q}\right]_{\gamma_{p c, q}}=B_{f, q}
$$

where $B_{f}$ is a surface field that defines the flux density discontinuity. But, as stated in (11), the total solution has no $B_{f}$,

$$
B_{f}=B_{f, p}+B_{f, q}=0 \text { so, } B_{f, q}=-B_{f, p}
$$

consequently,

$$
B_{f, q}=-\left[\mathbf{n} \cdot \mathbf{B}_{p}\right]_{\gamma_{p c, p}}
$$

As the internal field of $\Omega_{p c}$ is null, this discontinuity is treated as a boundary condition,

$$
\left.\mathbf{n} \cdot \mathbf{B}_{q}\right|_{\gamma_{p c}}=-\left.\mathbf{n} \cdot \mathbf{B}_{p}\right|_{\gamma_{p c}}
$$

As the field $\mathbf{B}_{p}$ is only necessary along $\gamma_{p c}$, the BiotSavart field is only solved along this boundary in problem $p$, reducing the computational time of the entire problem. The total solution, $p+q$, can also be interpreted as the Dirichlet boundary condition, i.e., $\left.\mathbf{n} \cdot \mathbf{B}\right|_{\gamma_{p c}}=0$, which imposes a zero normal component on the fields, offering a solution for further comparisons.

\section{Volume source}

As previously mentioned, the aim of this work is to take advantage of the SPM in order to reduce the domain where the Biot-Savart field is obtained and this is achieved by considering volume sources only along the active parts of this domain.

So, given a first problem $p$, with its constitutive relation and Maxwell equations,

$$
\begin{gathered}
\mathbf{H}_{p}=\frac{1}{\mu_{p}} \mathbf{B}_{p} \\
\operatorname{rot} \mathbf{H}_{p}=\mathbf{J} \\
\operatorname{div} \mathbf{B}_{p}=0
\end{gathered}
$$

it is possible to perturb this problem changing the magnetic permeability of a certain region, from $\mu_{p}$ to $\mu_{q}$, where $q$ is a subsequent problem, resulting in a new constitutive relation for the total problem $p+q$,

$$
\mathbf{H}_{p}+\mathbf{H}_{q}=\frac{1}{\mu_{q}}\left(\mathbf{B}_{p}+\mathbf{B}_{q}\right)
$$

The difference between the solutions (29) and (32) gives

$$
\mathbf{H}_{q}=\left(\frac{1}{\mu_{q}}-\frac{1}{\mu_{p}}\right) \mathbf{B}_{p}+\frac{1}{\mu_{q}} \mathbf{B}_{q}
$$

and suggests that problem $q$ depends on the solution of problem $p$ only along those perturbed regions, from $\mu_{p}$ to $\mu_{q}$, i.e., the active regions. Moreover, the total solution should satisfy the Ampère equation,

$$
\operatorname{rot}\left(\mathbf{H}_{p}+\mathbf{H}_{q}\right)=\mathbf{J}
$$

where it is possible to observe that $\mathbf{H}_{q}$ is curl-free. Applying the Gauss equation,

$$
\operatorname{div}\left(\mathbf{B}_{p}+\mathbf{B}_{q}\right)=0
$$

one can see that $\mathbf{B}_{q}$ is divergence-free. This means that the field $\mathbf{H}_{q}$ has the same behavior of the field generated by a permanent magnet, $\mathbf{H}_{c}$ in (4), and allows to take it into account in the $\mathbf{B}_{r}$ term of the FFEM magnetostatic formulation given in (15), thanks to (3) and (4).

Finally, re-writing (33) and defining the total field of the second problem in terms of the source and reaction fields, $\mathbf{H}_{q}=\mathbf{H}_{q s}+\mathbf{H}_{q r}$ (11), one obtains an equation with the same 
shape of (14), which consequently can be solved through the FFEM,

$$
\frac{1}{\mu_{q}} \mathbf{B}_{q}+\operatorname{grad} \psi_{q}=\mathbf{H}_{q s}-\left(\frac{1}{\mu_{q}}-\frac{1}{\mu_{p}}\right) \mu_{0} \mathbf{H}_{p}
$$

and contains the volume source term,

$$
\mathbf{V S}=-\left(\frac{1}{\mu_{q}}-\frac{1}{\mu_{p}}\right) \mu_{0} \mathbf{H}_{p}
$$

The field $\mathbf{H}_{q s}$ is zero because this problem does not consider the source field but only the volume sources.

As the perturbation regards the change from $\mu_{p}$ to $\mu_{q}$, there would have no problem to consider non-linear magnetic materials, once $\mu_{q}$ can be considered as element based.

If the model contains external reluctances with $\mathrm{mmf}$ sources, simplifying possible windings, they are not taken into account in the discretized domain. These $m m f$ sources need to be corrected using the factor given in (37).

Reference [22] presents a way to correct problem $q$ for higher values of $\mu_{q}$, i.e., $\mathbf{H}_{p}$ approaches to $-\mathbf{H}_{q}$. This correction is important in the case of projections of curl $\mathbf{A}$ [23] between different meshes and that is not the case here because the $\mathbf{H}_{p}$ field is calculated directly along the mesh used to solve problem $q$.

\section{Applications}

In order to validate the proposed methodology, some test cases are presented following the order of complexity. First, the correction for the Biot-Savart boundary condition is solved together with a perfect conductor simplification on a simple geometry. Then, one 3-D magnetic device is completely solved using this proposed methodology. Finally, this device is simplified using the FFEM+RNM coupling, as proposed in [3], and solved considering the subproblem technique.

The geometries and meshes are generated with the Gmsh program [24] and the A-formulation is solved using the program GetDP, which abbreviation means a "General Environment for the Treatment of Discrete Problems" [25]-[27].

\section{A. Correction of the boundary condition not imposed by Biot-Savart}

These preliminary test cases are based on a 3-D geometry obtained by the extrusion of a 2-D model, composed of two conductors and a magnetic core, as depicted in Fig. 4a. The top view of the 3-D mesh generated is shown in Fig. 4b. One horizontal probe line placed in the center of the model is used to compare the results locally.

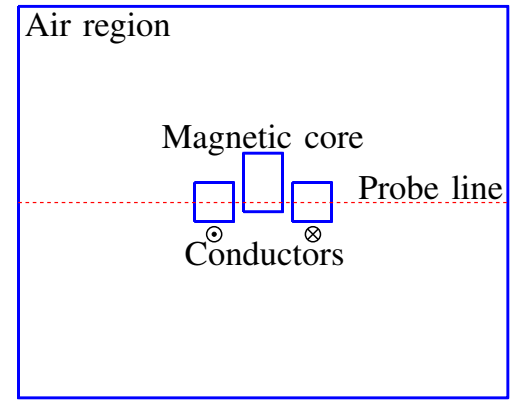

(a) 2-D geometry (extruded to build a 3-D mesh).

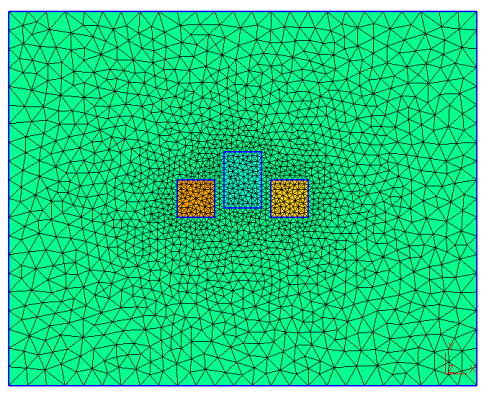

(b) Top view of the 3-D mesh.

Fig. 4. Geometry and mesh of the extruded domain.

In order to show the correction effect solely, the normal component of the magnetic field is obtained along the external surface with Biot-Savart, (Fig. 5), and the relative permeability of the magnetic core is set as unitary.

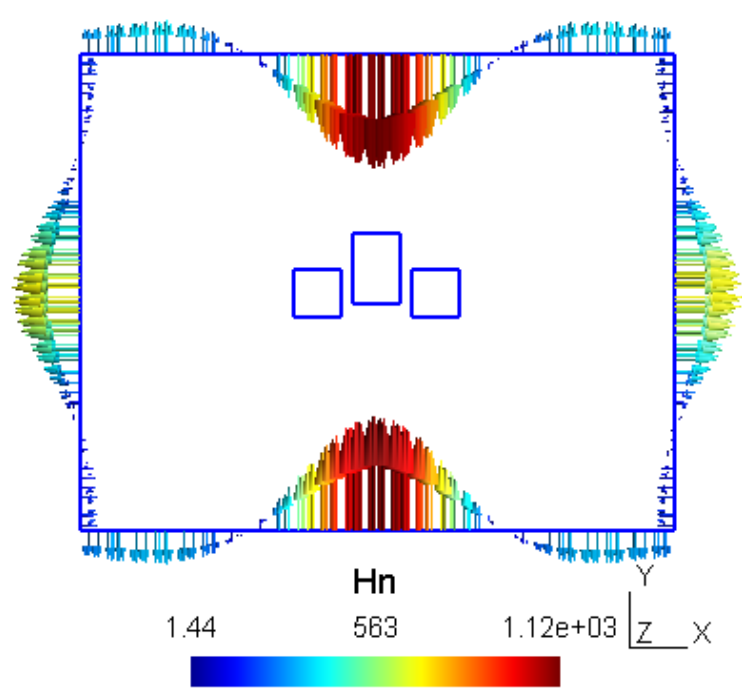

Fig. 5. Magnetic field obtained with Biot-Savart along the $\Gamma$.

The problem is solved considering the correction presented in Section III-B and the correction field obtained is shown in Fig. 6. The color scale is set as logarithmic in order to show a wider range of field magnitudes. 


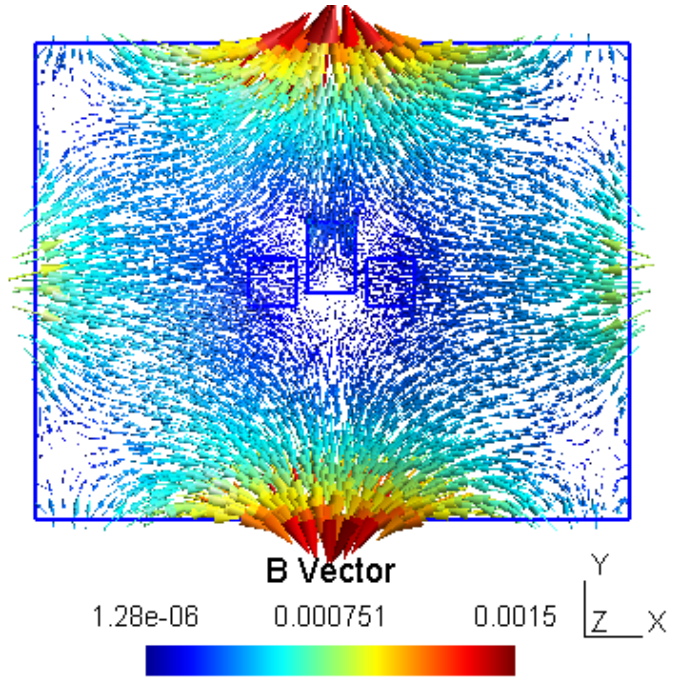

Fig. 6. Flux density distribution along $\Omega$.

As defined in Section III-B, the direction of the fields $\mathbf{H}$, Fig. 5, and B, Fig. 6, have opposite directions along the outer surface $\Gamma$, where the correction is applied.

\section{B. Perfect conductor boundary condition}

The magnetic core is now considered as a perfect conductor, $\Omega_{p c}$, and the correction presented in Section III-C is applied along its boundary. The Biot-Savart correction is also considered.

The correction field is shown in Fig. 7 and the comparison with the classical FEM along the probe line is presented in Fig. 8, where $\mathbf{B}_{p}(B S)$ is the source field obtained with BiotSavart, $\mathbf{B}_{q}$ is the correction field and $\mathbf{B}_{q}+\mathbf{B}_{p}$ is the sum of both quantities.

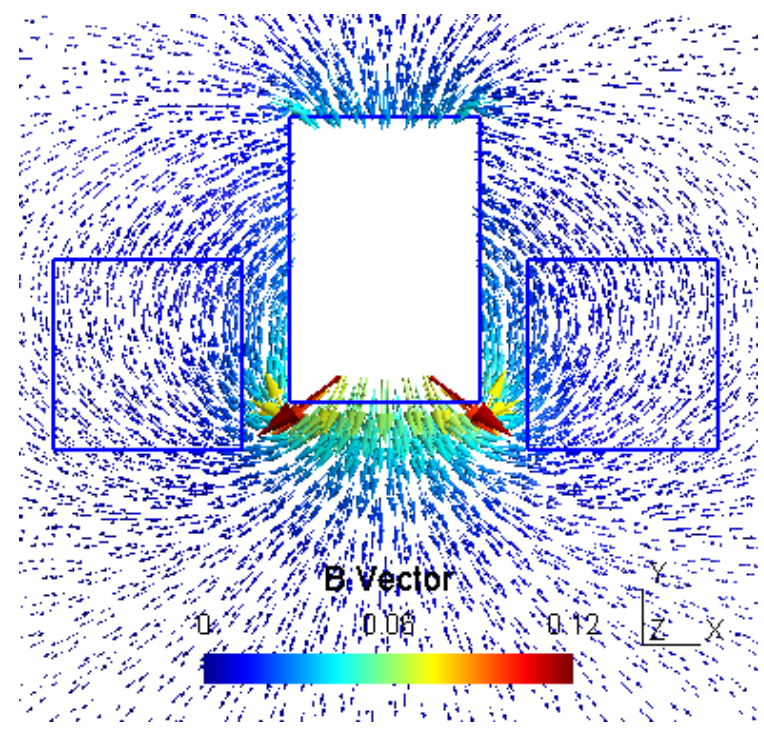

Fig. 7. Flux density distribution - problem $q$.

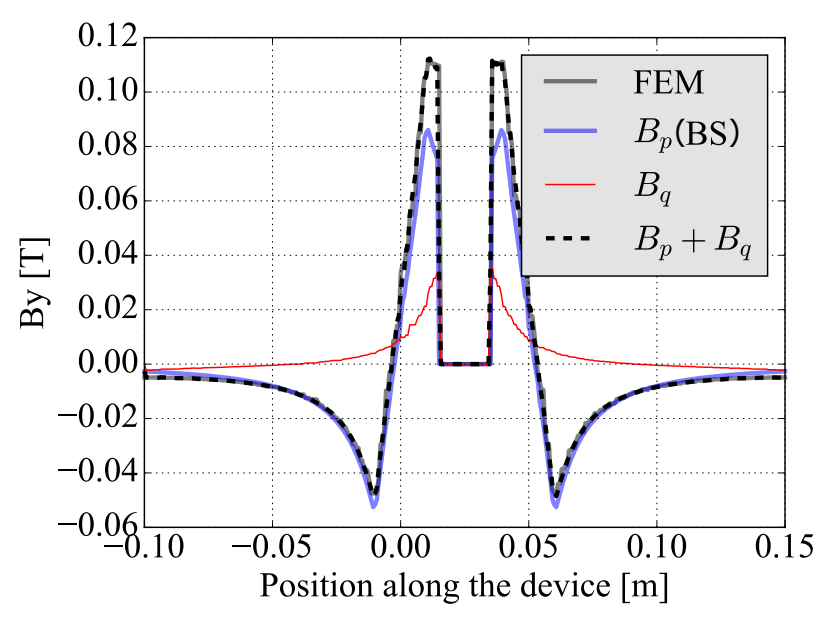

Fig. 8. Field $B_{y}$ along the probe line.

Fig. 9 shows a close view of By.

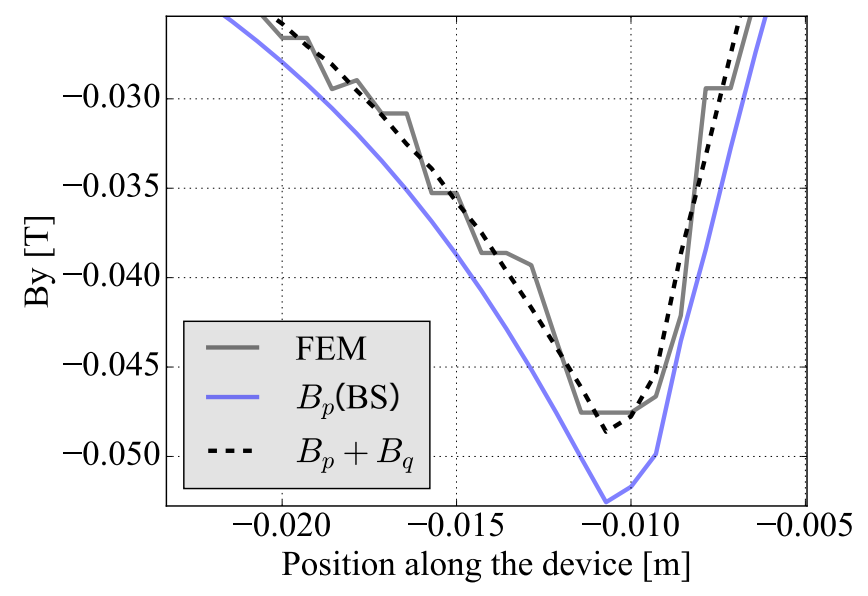

Fig. 9. Field $B_{y}$ along the probe line - zoom.

One can observe that the field obtained from Biot-Savart, $B_{p}(B S)$, is a continuous solution and when it is summed up to the one obtained with a first order FFEM, the total solution, i.e., $\mathbf{B}_{q}+\mathbf{B}_{p}$ becomes more continuous. Since this is not the case for the FFEM solution, this fact can be interpreted as a gain of accuracy of the proposed method.

\section{Volume sources}

Now, a more realistic magnetic device is simulated. As this work is a sequence of [3], to make the computational time comparison based on the conventional FFEM easier and clearer, the magnetic device presented in [3] is utilized here as well. Furthermore, its vertical windings close to the air gaps generate higher magnetic fields along these regions, consequently, increasing the problem $p$ contribution in the total solution, which is an important aspect due to the different behaviors of both solutions.

It is composed by two windings fed in opposite directions by a current density of $2.7310^{7} \mathrm{~A} / \mathrm{m}^{2}$ and two magnetic regions, with $\mu=2000 \mu_{0}$, separated by an air gap, as shown in Fig. 
10. The depth of the magnetic parts, air gap regions, and the outer air domain are 20,50 and $60 \mathrm{~mm}$, respectively.

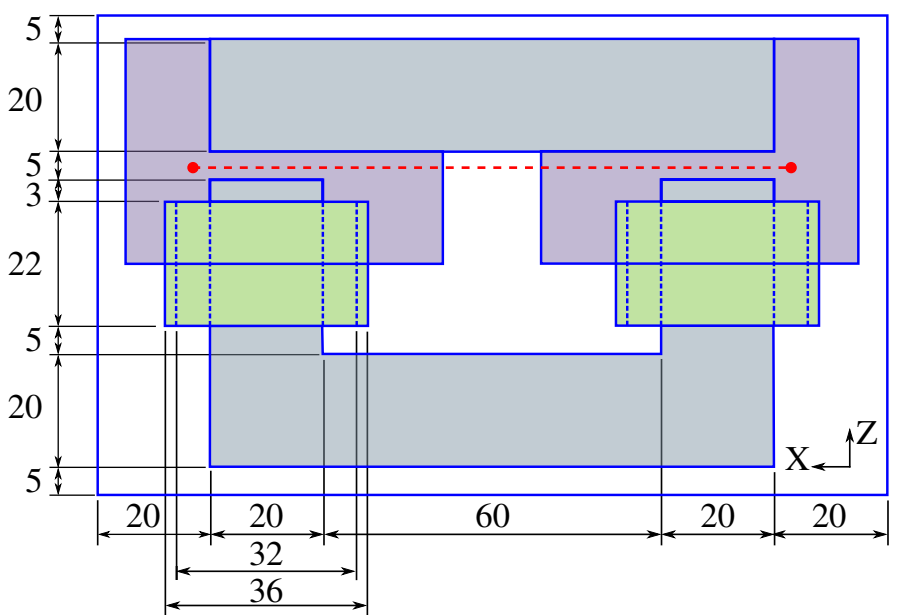

$\square$ Windings

$\square$ Magnetic parts

Air gap regions

-- Probe line

All dimensions are in $\mathrm{mm}$.

Fig. 10. Geometry of the 3-D device.

A tetrahedral mesh of the active parts and windings is shown in Fig. 11.

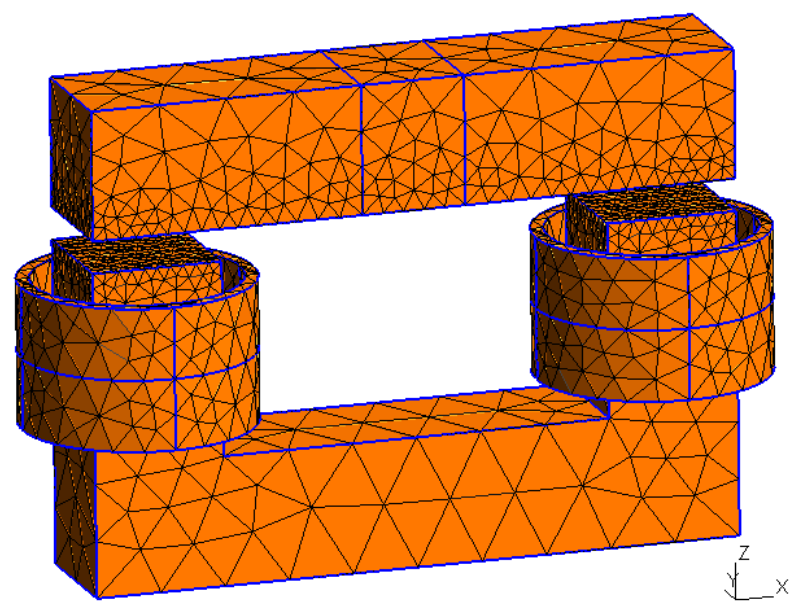

Fig. 11. 3-D mesh of the device.

This magnetic device is completely solved using the volume source described in Section III-D, and the Biot-Savart correction defined in Section III-B.

The field $\mathbf{H}_{p}$ presented in Fig. 12 was obtained by BiotSavart only along the active parts, reducing the computational time to $34 \%$ compared to the computational time of the classical solution, where Biot-Savart field is needed along the entire domain.

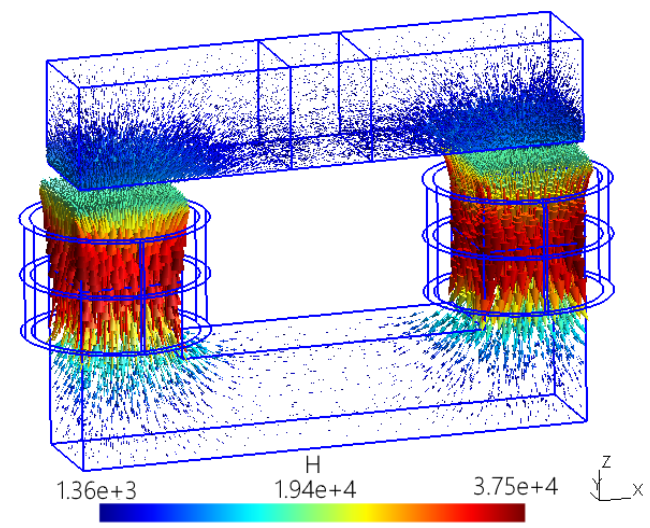

Fig. 12. Source field $\mathbf{H}_{p}$ obtained only along the active parts.

Then, the problem $q$ solution is obtained solving (36) with FFEM (19), considering the source field $\mathbf{H}_{p}$ given in Fig. 12 as a volume source, (37).

Even if not necessary to calculate the $p$ problem solution along the entire domain, but only along the interest regions, it is shown in Fig. 13a as a post-processing task. Therefore, the total $(p+q)$ solution of this problem is represented by the sum of these fields (Fig. 13a $+13 b$ ), where Fig. 13b shows the solution of problem $q$.

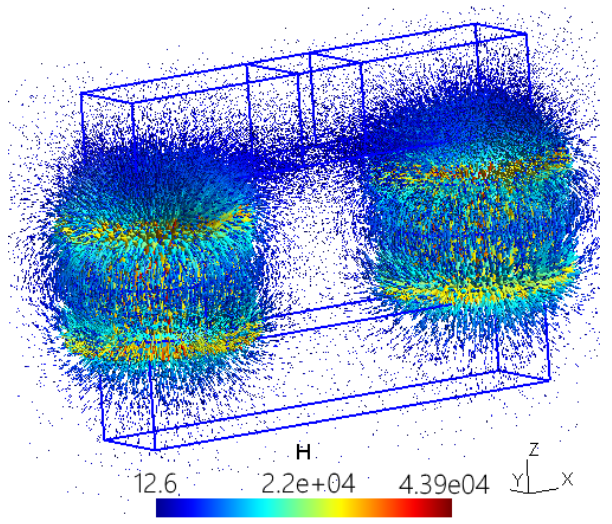

(a) Magnetic field $\mathbf{H}_{p}$ obtained with BS. $\mathbf{B}_{p}=$ $\mu_{0} \mathbf{H}_{p}$.

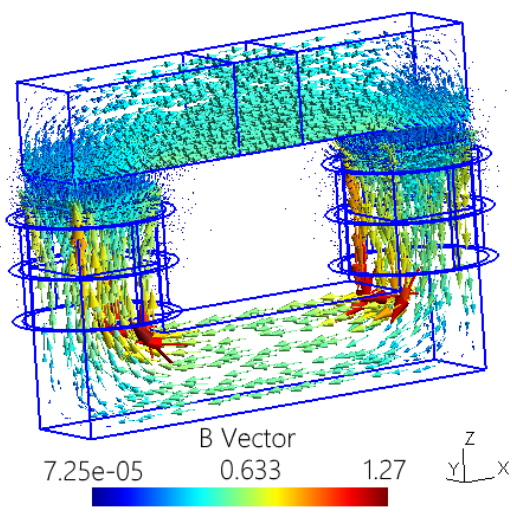

(b) Flux density $\mathbf{B}_{q}$ obtained with FFEM.

Fig. 13. Field maps representing the solutions of the problems $p$ and $q$ for the complete model. 
A comparison between the total solution, $\mathbf{B}_{p}+\mathbf{B}_{q}$, and the results obtained with the classical FFEM along the probe line is given in Fig. 14. It is important to mention that the problem $p$ consists of calculating the Biot-Savart field along this line, as a post-processing operation.

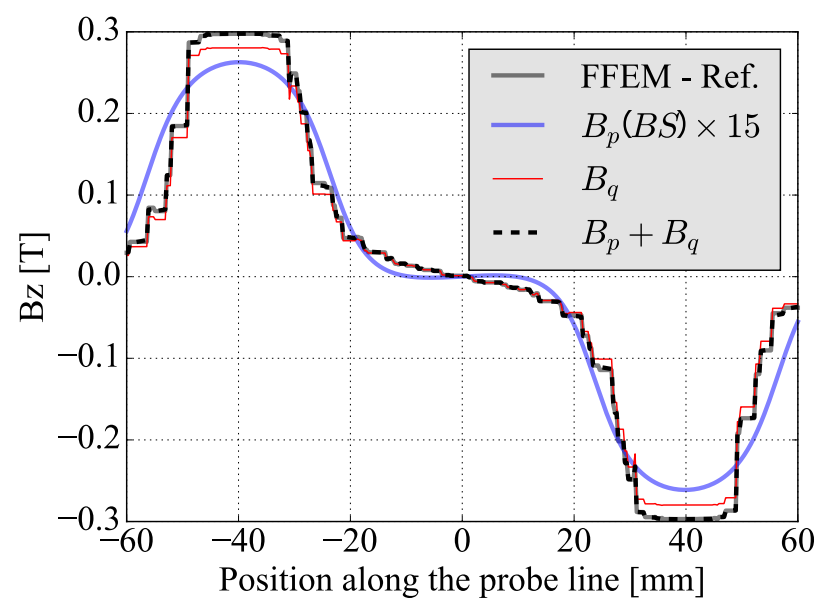

Fig. 14. Field $B_{z}$ along the air region.

\section{Volume sources + Coupling $($ FFEM + RNM $)$}

The model presented in Fig. 10 is simplified as shown in Fig. 15, where half of the windings are inside the meshed domains and the other halves of the Ampere-turns are considered as $m m f$ sources in the external circuit.

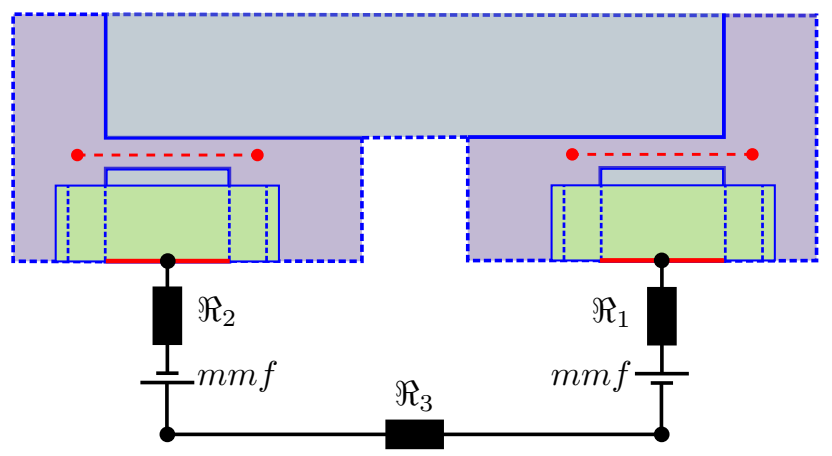

Fig. 15. Coupling between FFEM and RNM.

The source field, $\mathbf{H}_{p}$, is obtained considering the Biot-Savart integration along the complete windings only along the active parts of the meshed domain, reducing the computational time needed for the source field calculation to about $12 \%$ of the computational time of the classical method.

Finally, the volume source (37) is used as a source term in the FFEM system and its correction is also applied in the external $m m f$ sources. So, this coupled problem is solved using also the subproblem technique and the flux density distribution obtained is presented in Fig. 16b. The problem $p$ solution is shown in Fig. 16a, only with the visualization purpose as already mentioned, composing the total solution (Fig. 16a + Fig. 16b)

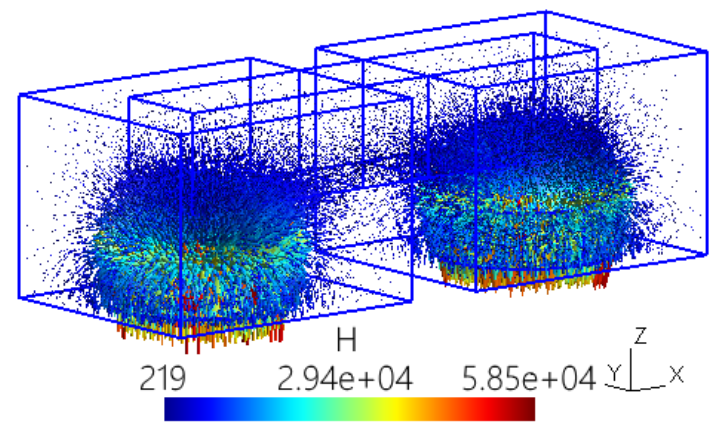

(a) Magnetic field $\mathbf{H}_{p}$ obtained with Biot-Savart. $\mathbf{B}_{p}=\mu_{0} \mathbf{H}_{p}$.

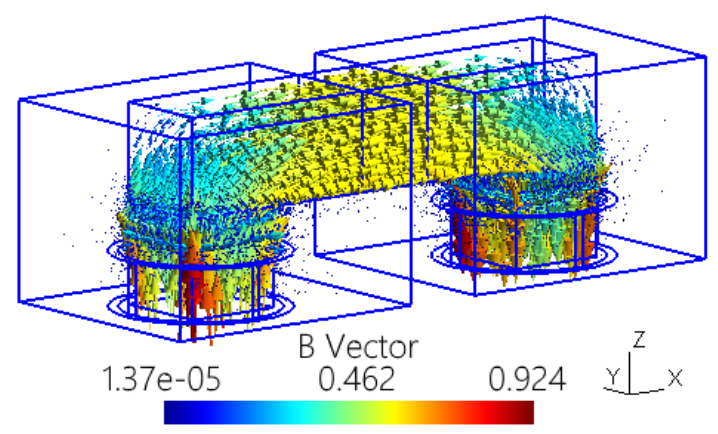

(b) Flux density $\mathbf{B}_{q}$ obtained with FFEM.

Fig. 16. Field maps representing the solutions of the problems $p$ and $q$ for the reduced model.

Then, this coupled model is also solved using the classical coupling [3] and the comparison along the probe line is presented in Fig. 17.

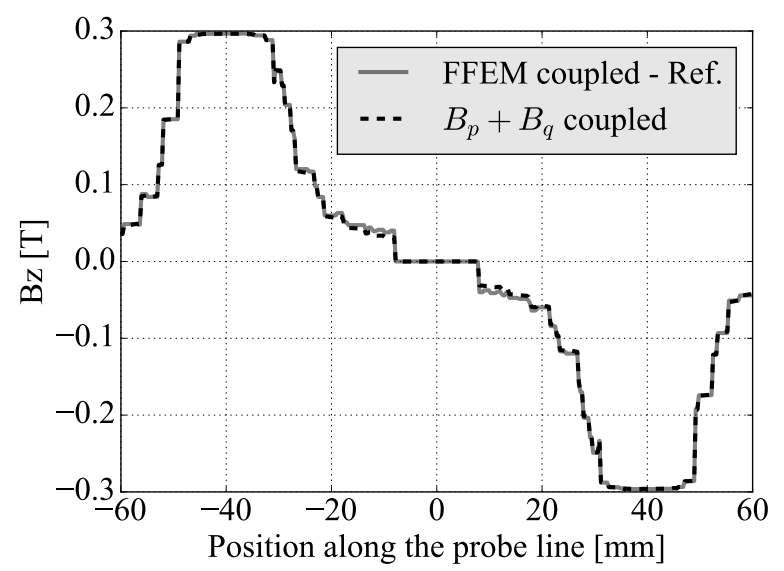

Fig. 17. Field $B_{z}$ along the probe line.

\section{E. Time comparison of the source field calculation}

In reference [3], the source field is computed along the entire domains, but in this work it is calculated only along the ferromagnetic parts. As the test cases are the same for both works, it is possible to compare the simulation time necessary to obtain the source field in all those test cases. The comparison is presented in Table I, where the percentage 
values refer to the computational time necessary to obtain the source field along the complete model (indicated with "Ref." in Table I) presented in [3].

TABLE I

COMPARISON OF THE SIMULATION TIME IN RELATION WITH THE COMPLETE MODEL SOLVED WITHOUT THE SPM.

\begin{tabular}{ccc}
\hline \hline Model & $\begin{array}{c}\text { Without the SPM } \\
\text { (From reference [3]) }\end{array}$ & $\begin{array}{c}\text { With the SPM } \\
\text { (Proposed method) }\end{array}$ \\
\hline Complete & Ref. & $33.6 \%$ \\
Coupled (FFEM+RNM) & $52.2 \%$ & $12.1 \%$ \\
\hline \hline
\end{tabular}

Notwithstanding the coupling FFEM+RNM can save computational effort and time, the results obtained in this work make evident how the subproblem technique can represent an important speedup.

\section{CONCLUSION}

The proposed methodology allowed to reduce the computational time to obtain the source field required in the Facet Finite Element Method by applying subproblem technique. It was based on the application of the volume source together with a correction for the boundary condition not imposed by Biot-Savart but imposed by the FEM technique, allowing a direct superposition of those two problems, $p$ and $q$. Computationally speaking, it was possible to find that this correction is not a time consuming task. However, it can represent a significant impact on the accuracy of solution, mainly near the outer surfaces of the domain.

The solutions were presented in the order of complexity, showing the different approaches independently, which can be helpful to apply in others situations. The results obtained for one simple and other complex cases were compared with the results calculated through the classical FEM (A-formulation) and FFEM, presenting an acceptable accuracy.

For the real case studied, it was possible to reduce the Biot-Savart computational time to $12 \%$ if compared with the classical method. As this factor is directly dependent on the volume ratio between the magnetic and non-magnetic regions in the domain, it can vary considerably. Nonetheless, normally the 3-D cases demands a considerable large air regions due to the imposition of the $\left.\mathbf{n} \cdot \mathbf{B}\right|_{\Gamma_{B}}=0$ boundary condition, this factor tends to be small in the majority of practical cases.

\section{ACKNOWLEDGMENT}

This work is supported by CNPq (Conselho Nacional de Desenvolvimento Científico e Tecnológico - Brazil), project 200215/2016-7.

\section{REFERENCES}

[1] A. S. Nunes, O. Chadebec, G. Meunier, and P. Kuo-peng, "Magnetic devices analysis by Face FEM coupled with standard reluctance network," in 20 h Compumag Conference on the Computation of Electromagnetic Fields, vol. 41, no. 5. Montréal: IEEE, 2015, pp. 1-2.

[2] A. S. Nunes, O. Chadebec, P. Kuo-Peng, P. Dular, and G. Meunier, "3D magnetic devices analysis using facet FEM formulation coupled with reluctance network method," in 2016 IEEE Conference on Electromagnetic Field Computation (CEFC). Miami, FL: IEEE, nov 2016.
[3] A. S. Nunes, O. Chadebec, P. Kuo-Peng, P. Dular, and G. Meunier, "A coupling between the facet finite element and reluctance network methods in 3-D," IEEE Transactions on Magnetics, vol. 53, no. 10, pp. $1-10$, oct 2017.

[4] A. Demenko, "Network models of three-dimensional electromagnetic fields," in IET 7th International Conference on Computation in Electromagnetics (CEM 2008). IEE, 2008, pp. 70-80. [Online]. Available: http://digital-library.theiet.org/content/conferences/10.1049/ cp_20080226

[5] A. Demenko and J. K. Sykulski, "Analogies between finite-difference and finite-element methods for scalar and vector potential formulations in magnetic field calculations," IEEE Transactions on Magnetics, vol. 52, no. 6, pp. 1-6, jun 2016.

[6] R. M. Wojciechowski and C. Jedryczka, "Description of the windings of the electromagnetic energy converters using the modified T0 method," in 2017 18th International Symposium on Electromagnetic Fields in Mechatronics, Electrical and Electronic Engineering (ISEF) Book of Abstracts. IEEE, Sep. 2017.

[7] P. Dular, R. V. Sabariego, and L. Krähenbühl, "Subdomain perturbation finite element method for skin and proximity effects in inductors," COMPEL - The international journal for computation and mathematics in electrical and electronic engineering, vol. 27, no. 1, pp. 72-84, 2008. [Online]. Available: http://www.emeraldinsight.com/doi/abs/10. $1108 / 03321640810836654$

[8] Z. Badics, Y. Matsumoto, K. Aoki, F. Nakayasu, M. Uesaka, and K. Miya, "An effective 3-D finite element scheme for computing electromagnetic field distortions due to defects in eddycurrent nondestructive evaluation," IEEE Transactions on Magnetics, vol. 33, no. 2, pp. 1012-1020, 1997. [Online]. Available: http: //dx.doi.org/10.1109/20.558521

[9] P. Dular, R. V. Sabariego, M. V. Ferreira Da Luz, P. Kuo-Peng, and L. Krähenbühl, "Perturbation finite element method for magnetic model refinement of air gaps and leakage fluxes," IEEE Transactions on Magnetics, vol. 45, no. 3, pp. 1400-1403, 2009.

[10] P. Dular, R. V. Sabariego, J. Gyselinck, and L. Krähenbühl, "Subdomain finite element method for efficiently considering strong skin and proximity effects," COMPEL - The international journal for computation and mathematics in electrical and electronic engineering, vol. 26, no. 4, pp. 974-985, Aug. 2007

[11] P. Dular, R. Sabariego, and L. Krahenbuhl, "Subdomain perturbation finite-element method for skin and proximity effects," IEEE Transactions on Magnetics, vol. 44, no. 6, pp. 738-741, Jun. 2008. [Online]. Available: http://dx.doi.org/10.1109/TMAG.2007.915817

[12] P. Dular, R. V. Sabariego, and L. Krähenbühl, "Magnetic model refinement via a perturbation finite element method from $1 \mathrm{D}$ to $3 \mathrm{D}$," COMPEL: The International Journal for Computation and Mathematics in Electrical and Electronic Engineering, vol. 28, no. 4, pp. 974-988, Jul. 2009. [Online]. Available: http://www.emeraldinsight.com/10.1108/ 03321640910959044

[13] P. Dular, L. Krähenbühl, M. V. F. da Luz, P. Kuo-Peng, and C. Geuzaine, "Progressive inductor modeling via a finite element subproblem method," COMPEL, vol. 34, no. 3, pp. 851-863, May 2015. [Online]. Available: http://dx.doi.org/10.1108/COMPEL-10-2014-0279

[14] P. Dular, V. Peron, L. Krahenbuhl, and C. Geuzaine, "Subproblem finite-element refinement of inductors from wire to static and dynamic volume models," IEEE Trans. Magn., vol. 51, no. 3, pp. 1-4, Mar. 2015. [Online]. Available: http://dx.doi.org/10.1109/TMAG.2014.2360232

[15] P. Dular, P. Kuo-Peng, M. V. F. da Luz, and L. Krahenbuhl, "Progressive current source models in magnetic vector potential finite-element formulations," IEEE Trans. Magn., vol. 52, no. 3, pp. 1-4, Mar. 2016. [Online]. Available: http://hdl.handle.net/2268/191454

[16] M. Boutaayamou, R. V. Sabariego, and P. Dular, "A perturbation method for the 3D finite element modeling of electrostatically driven MEMS," 2007. [Online]. Available: http://dx.doi.org/10.1109/ESIME. 2007.359942

[17] P. Dular, V. Pèron, L. Krähenbühl, and C. Geuzaine, "Progressive eddy current modeling via a finite element subproblem method," International Journal of Applied Electromagnetics and Mechanics, vol. 46, no. 2, pp. 341-348, 2014.

[18] P. Raviart and J. Thomas, "A mixed finite element method for 2-nd order elliptic problems," in Mathematical aspects of finite element methods, I. Galligani and E. Magenes, Eds., vol. 606. Rome: Springer, Dec. 1977, pp. 292-315.

[19] A. Bossavit, "A rationale for "edge-elements" in 3-D fields computations," IEEE Transactions on Magnetics, vol. 24, no. 1, pp. 74-79, 1988

[20] A. Demenko and J. Sykulski, "Network equivalents of nodal and edge 
elements in electromagnetics," IEEE Transactions on Magnetics, vol. 38, no. 2, pp. 1305-1308, Mar. 2002.

[21] A. Demenko and J. Sykulski, "Magneto-electric network models in electromagnetism," COMPEL - The international journal for computation and mathematics in electrical and electronic engineering, vol. 25, no. 3 , pp. 581-588, jul 2006.

[22] P. Dular, R. V. Sabariego, C. Geuzaine, M. V. Ferreira da Luz, P. Kuo-Peng, and L. Krahenbuhl, "Finite Element Magnetic Models via a Coupling of Subproblems of Lower Dimensions," IEEE Transactions on Magnetics, vol. 46, no. 8, pp. 2827-2830, Aug. 2010. [Online]. Available: http://ieeexplore.ieee.org/lpdocs/epic03/ wrapper.htm?arnumber $=5512990$

[23] C. Geuzaine, B. Meys, F. Henrotte, P. Dular, and W. Legros, "A galerkin projection method for mixed finite elements," IEEE Trans. Magn., vol. 35, no. 3, pp. 1438-1441, May 1999. [Online]. Available: http://dx.doi.org/10.1109/20.767236

[24] C. Geuzaine and J.-F. Remacle, "Gmsh: A 3-d finite element mesh generator with built-in pre- and post-processing facilities," International Journal for Numerical Methods in Engineering, vol. 79, no. 11, pp. 1309-1331, sep 2009.

[25] P. Dular, C. Geuzaine, F. Henrotte, and W. Legros, "A general environment for the treatment of discrete problems and its application to the finite element method," IEEE Transactions on Magnetics, vol. 34, no. 5, pp. 3395-3398, Sep. 1998.

[26] P. Dular, C. Geuzaine, A. Genon, and W. Legros, "An evolutive software environment for teaching finite element methods in electromagnetism," IEEE Transactions on Magnetics, vol. 35, no. 3, pp. 1682-1685, May 1999.

[27] C. Geuzaine, "GetDP: a general finite-element solver for the de Rham complex," in PAMM Volume 7 Issue 1. Special Issue: Sixth International Congress on Industrial Applied Mathematics (ICIAM07) and GAMM Annual Meeting, Zürich 2007, vol. 7. Wiley, 2008, pp. $1010603-$ 1010604 . 